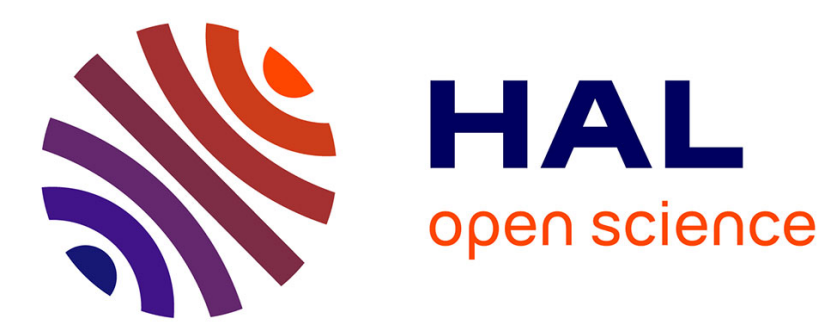

\title{
Reusing Legacy DSLs with Melange
}

Thomas Degueule, Benoit Combemale, Arnaud Blouin, Olivier Barais

\section{To cite this version:}

Thomas Degueule, Benoit Combemale, Arnaud Blouin, Olivier Barais. Reusing Legacy DSLs with Melange. 15th Workshop on Domain-Specific Modeling, Oct 2015, Pittsburgh, United States. hal$01197039 \mathrm{v} 2$

\section{HAL Id: hal-01197039 \\ https://inria.hal.science/hal-01197039v2}

Submitted on 1 Oct 2015

HAL is a multi-disciplinary open access archive for the deposit and dissemination of scientific research documents, whether they are published or not. The documents may come from teaching and research institutions in France or abroad, or from public or private research centers.
L'archive ouverte pluridisciplinaire HAL, est destinée au dépôt et à la diffusion de documents scientifiques de niveau recherche, publiés ou non, émanant des établissements d'enseignement et de recherche français ou étrangers, des laboratoires publics ou privés. 


\section{Reusing Legacy DSLs with Melange}

\author{
Thomas Degueule \\ Benoit Combemale \\ INRIA, France \\ firstname.lastname@inria.fr
}

\author{
Arnaud Blouin \\ INSA Rennes, France \\ arnaud.blouin@irisa.fr
}

\author{
Olivier Barais \\ University of Rennes 1, France \\ olivier.barais@irisa.fr
}

\begin{abstract}
The proliferation of independently-developed and constantlyevolving domain-specific languages (DSLs) in many domains raises new challenges for the software language engineering community. Instead of starting the definition of new DSLs from scratch, language designers would benefit from the reuse of previously defined DSLs. While the support for engineering isolated DSLs is getting more and more mature, there is still little support in language workbenches for importing, assembling, and customizing legacy languages to form new ones. Melange is a new language workbench where new DSLs are built by assembling pieces of syntax and semantics. These pieces can be imported and subsequently extended, restricted, or customized to fit specific requirements. The demonstration will introduce the audience to the main features of Melange through the definition of an executable DSL for the design and execution of Internet of Things systems. Specifically, we will show how such a language can be obtained from the assembly of other popular languages while maintaining the compatibility with their tools and transformations.
\end{abstract}

Categories and Subject Descriptors D3.2 [Language Classifications]: Specialized application languages

Keywords Domain-specific languages, language workbench, language reuse, model typing, melange

\section{Introduction}

Domain-specific languages (DSLs) are increasingly used to handle specific concerns in the development of complex software systems [1]. However, developing a DSL is still a costly and time-consuming task that requires advanced skills in language design: language designers have to specify the abstract syntax of their languages, their concrete syntax, semantics, and tooling (e.g. editors, checkers, code generators, etc.). Language workbenches assist language designers by providing the right tools and methods to tame the complexity of language design and reduce the development costs [2]. While current workbenches (e.g. MetaEdit+, Spoofax, MPS, Xtext - to cite just a few) propose a diffuse way to reuse language modules, there is currently little support for assembling languages with customization facilities. Yet, it is likely that the creation of new DSLs could benefit from the efforts spent on the development of other ones, especially when their domains overlap. A mere example is the family of statecharts languages which, despite their specificities, share many similarities in their syntax and semantics [3]. The expected outcomes are twofold: one would like to import the definition of legacy language artifacts to engineer a new one while ensuring the compatibility with the tools and transformations defined on its ancestors. Of course, imported artifacts may not fit exactly the designer's expectations. It follows that support for language extension, restriction, and customization is required to tune them finely.

This paper is organized as follows. In Section 2, we introduce Melange, a new language workbench attempting to address each of these challenges. In Section 3, we present an outline of the proposed demonstration.

\section{The Melange Workbench}

Melange [4] is an open-source language workbench built on top of the Eclipse Modeling Framework (EMF) and tightly integrated with its ecosystem ${ }^{1}$. Thanks to the success of EMF in both academia and industry, this enables Melange's users to import and manipulate a wide spectrum of existing DSLs. In Melange, the abstract syntax of DSLs is defined in the form of a metamodel with the Ecore formalism. Their operational semantics is specified using the Xtend programming language ${ }^{2}$. More precisely, Melange supports the definition of aspects which allows to define the operational semantics of the concepts contained in a metamodel in a non-intrusive manner, based on static introduction [5]. In Melange, pieces of syntax and semantics can be imported and assembled to form new languages. The resulting languages follow the same design principles: they consist of a metamodel and a set of aspects that can be directly bundled and deployed as is, or reused in other assemblies. In order to fit unforeseen requirements or new environments, Melange also provides customization operators: languages can be merged together, inherited, or sliced. Each of these operators takes both syntax and semantics into account. The merge operator serves as a language unification mechanism and is inspired by the UML Package-

\footnotetext{
${ }^{1}$ http://melange-lang.org

${ }^{2}$ https://eclipse.org/xtend/
} 
Merge relation [6]. The slice operator is inspired by model slicing [7] and consists in extracting a subset of an existing language to be imported in a new one. Finally, the inherits operator allows to reuse the definition of one or more superlanguages into a new language. In addition to the merge operator, the inherits operator ensures that the resulting language remains compatible with its super-languages, i.e. the tools defined on a super-language can always be applied on its sub-language. Additionally, every language in Melange is associated with a structural interface captured in a model type [8]. This interface exposes the features that are publicly accessible on a language, $e$.g. its meta-classes, their structural features, and the methods defining its operational semantics. Most importantly, model types are linked one another with subtyping relations [9]. Intuitively, a model type is a subtype of another one if it exposes the exact same features, and possibly others, i.e. it is not any less capable. These relations and the associated type system provide model polymorphism, i.e. the possibility to manipulate a model through tools defined for different languages, providing that their interfaces match. Concretely, this means that when a language is built from another one (e.g. through inheritance or slicing), tools and transformations can be reused if the resulting interface remains substitutable with the one of its ancestor. The model polymorphism facility is not only available within Melange, but also contributed as a specialized resource management system directly in EMF. This means that any project relying on the EMF framework for model loading and serialization can benefit from the model polymorphism facilities provided by Melange. The overall approach is depicted in Figure 1.

\section{Demonstration Outline}

The demonstration will highlight the main features of Melange, illustrated through the creation of an executable DSL for the design and execution of Internet of Things (IoT) systems. The resulting IoT language is inspired from both general-purpose executable modeling languages such as fUML [10] and modeling languages dedicated to IoT such as ThingML [11]. We will show how the assembly operators of Melange foster the reuse of pre-existing languages. Specifically, the IoT language will be designed as an assembly of publicly-available DSLs: (i) an IDL language for specifying the structural interface of sensors (ii) Lua for expressing their behavior and (iii) an activity diagram to express concrete scenarios involving different sensors. Taken independently, each of these languages has been defined by different groups of people for specific purposes, unrelated to IoT systems. Combining them in a consistent way, however, leads to a new DSL particularly suited to a new context, i.e. the IoT domain. Because most of their syntax and semantics can be reused as is, this drastically reduce the development costs compared to a top-down approach. Of course, the syntax and semantics of two independent languages may not fit together perfectly when composed. Therefore, the demonstration will also focus

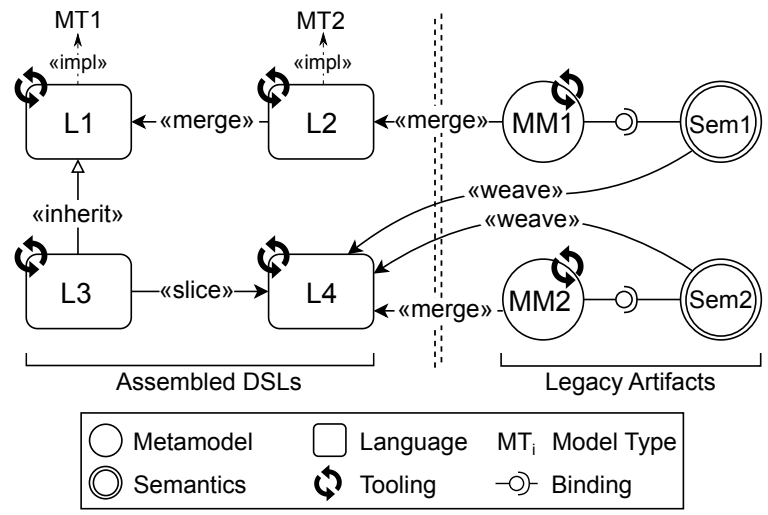

Figure 1: Assembling Languages using Melange. The right part depicts the legacy language artifacts (syntax or semantics) that must be assembled; the left part depicts the newly-created DSLs resulting from the assembly. Each language implements a specific set of model types which define how they are manipulated.

on the customization of these reused languages. Finally, the demonstration will illustrate how the resulting DSL remains compatible with the tools and transformations (e.g. checkers, editors) previously defined on the imported languages.

\section{References}

[1] Jon Whittle, John Hutchinson, and Mark Rouncefield. The state of practice in model-driven engineering. Software, IEEE, 31(3):79-85, 2014.

[2] Martin Fowler. Language workbenches: The killer-app for domain specific languages. 2005.

[3] Michelle L Crane and Juergen Dingel. UML vs. classical vs. Rhapsody statecharts: Not all models are created equal. In Model Driven Engineering Languages and Systems, pages 97-112. 2005.

[4] Thomas Degueule, Benoit Combemale, Arnaud Blouin, Olivier Barais, and Jean-Marc Jézéquel. Melange: A meta-language for modular and reusable development of dsls. In 8th International Conference on Software Language Engineering (SLE), 2015.

[5] Jean-Marc Jézéquel, Benoit Combemale, Olivier Barais, Martin Monperrus, and François Fouquet. Mashup of metalanguages and its implementation in the kermeta language workbench. Software \& Systems Modeling, pages 1-16, 2013.

[6] OMG. Unified Modeling Language 2.0, Infrastructure, 2005.

[7] Arnaud Blouin, Benoit Combemale, Benoit Baudry, and Olivier Beaudoux. Kompren: Modeling and generating model slicers. Software and Systems Modeling (SoSyM), pages 1-17, 2012.

[8] Jim Steel and Jean M. Jézéquel. On model typing. SoSyM, 6(4):401-413, 2007.

[9] Clément Guy, Benoît Combemale, Steven Derrien, Jim RH Steel, and Jean-Marc Jézéquel. On model subtyping. In Modelling Foundations and Applications, pages 400-415. Springer, 2012.

[10] OMG. Semantics of a foundational subset for executable UML models (FUML 1.0), 2011.

[11] Franck Fleurey, Brice Morin, Arnor Solberg, and Olivier Barais. MDE to manage communications with and between resourceconstrained systems. In Proc. of MODELS'11, pages 349-363, 2011. 


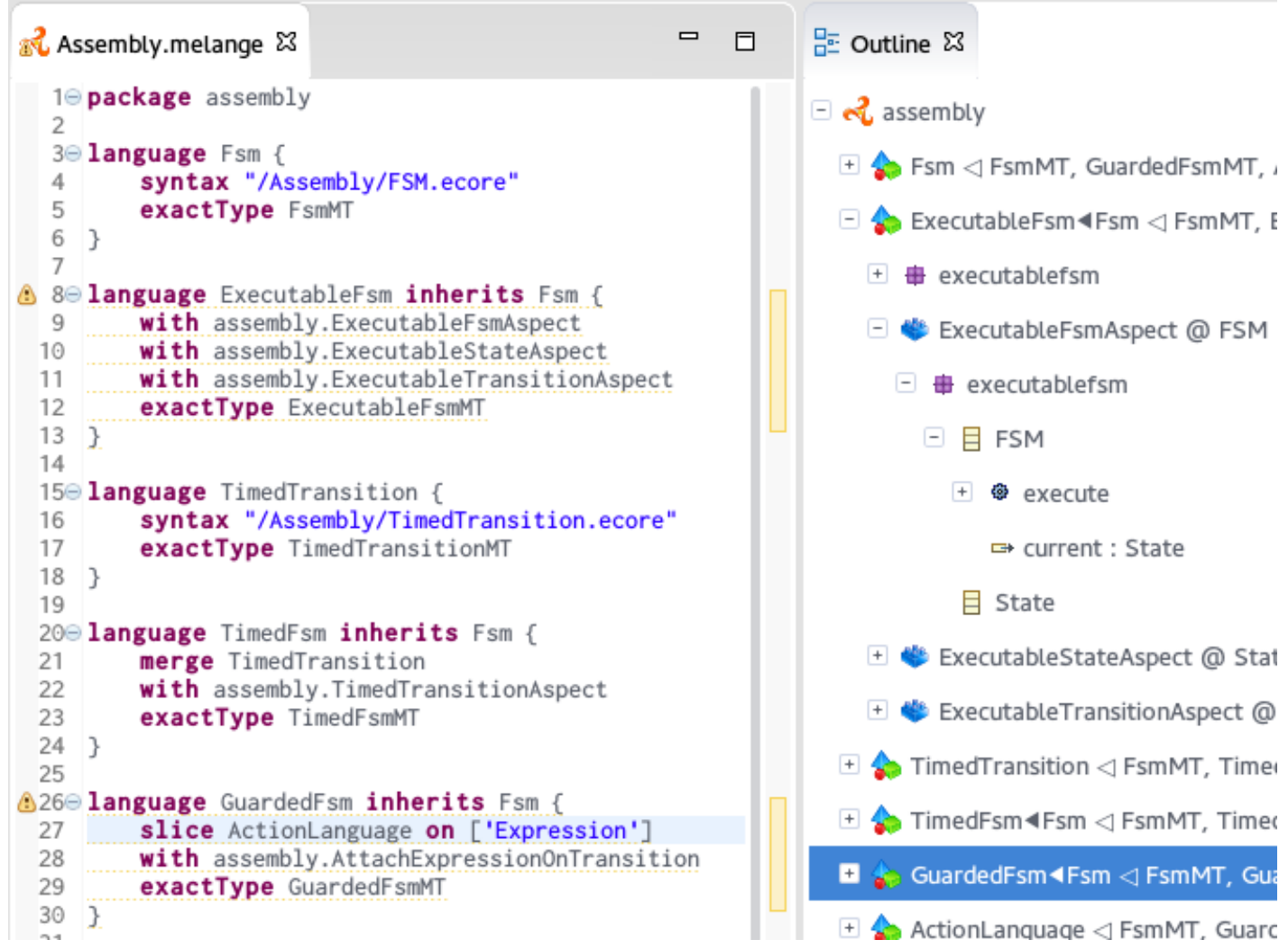

Figure 2: Assembling several variants of a finite-state machine language in Melange. The outline presents the abstract syntax of each language, the methods and runtime data inserted using aspects (e.g. a fire() method on transitions and the current state), and the subtyping relations linking their types. Language designers can thus build new languages on the one side and observe the results on the other side at the same time. 